\title{
The diagnosis of junior middle school mathematics learning disability:based on the Cognitive Diagnosis Theory
}

Fang Ping ${ }^{1}$ Wang Chao ${ }^{1}$ Jiang Yuan ${ }^{2}$

1 School of Psychology, Capital Normal University, Beijing ,China 100048 2 School of Psychology, Beijing Sport University, Beijing, China 100084

How to make accurate diagnosis for the mathematics learning disability have been the focus of psychology and education field for many years, The common diagnosis methods including The Grade-Level Inconsistent Model(GLIM), DN: CAS assessment tools, ERP technology and so on. GLIM and DN: CAS assessment tool are base on the Classical Test Theory(CTT), due to the deficit that CTT dealing with test error, these methods cann't provide a scientific result. Although the cognitive neuroscience methods such as ERP can provide a scientific result, they are not suitable for large-scale screening, and these methods can not ensure the accuracy of diagnosis as well as the efficiency. For this reason, this study propose to find a new way to screen junior middle school's children with mathematics learning disablity, in order to provide theoretical support for the future of mathematics learning difficulty research and practice, at first we analysed the literature in existing databases at home and abroad and build cognitive diagnosis attribute hierarchy model. We find out seven cognitive attribute to build attribute hierarchy model, they respectively are: attention maintain, working memory span, arithmetic ability, graphics perception, mathematics knowledge cognitive flexibility and strategy using, after determining the attribute hierarchy model, we compiled the 18 exam question, and gives its $Q$ matrix for the subsequent diagnostic tests and Model Fit Analysis.

Table1 Cognitive attribute of MLD in junior middle schoo

\begin{tabular}{|c|c|c|}
\hline dimensionality & factor & code \\
\hline attention & attention maintain & A1 \\
\hline Working Memory & $\begin{array}{c}\text { Working memory } \\
\text { span }\end{array}$ & A2 \\
\hline \multirow{2}{*}{$\begin{array}{c}\text { Math problem } \\
\text { solving ability }\end{array}$} & $\begin{array}{c}\text { grithmetic ability } \\
\text { perception }\end{array}$ & A3 \\
\cline { 2 - 3 } & $\begin{array}{c}\text { mathematics } \\
\text { knowledge }\end{array}$ & A5 \\
\hline \multirow{2}{*}{ Meta cognition } & cognitive flexibility & A6 \\
\cline { 2 - 3 } & strategy using & A7 \\
\hline
\end{tabular}

By considering the number of subjects, number of attributes and number of questions, we chose the DINA model for data analysis.

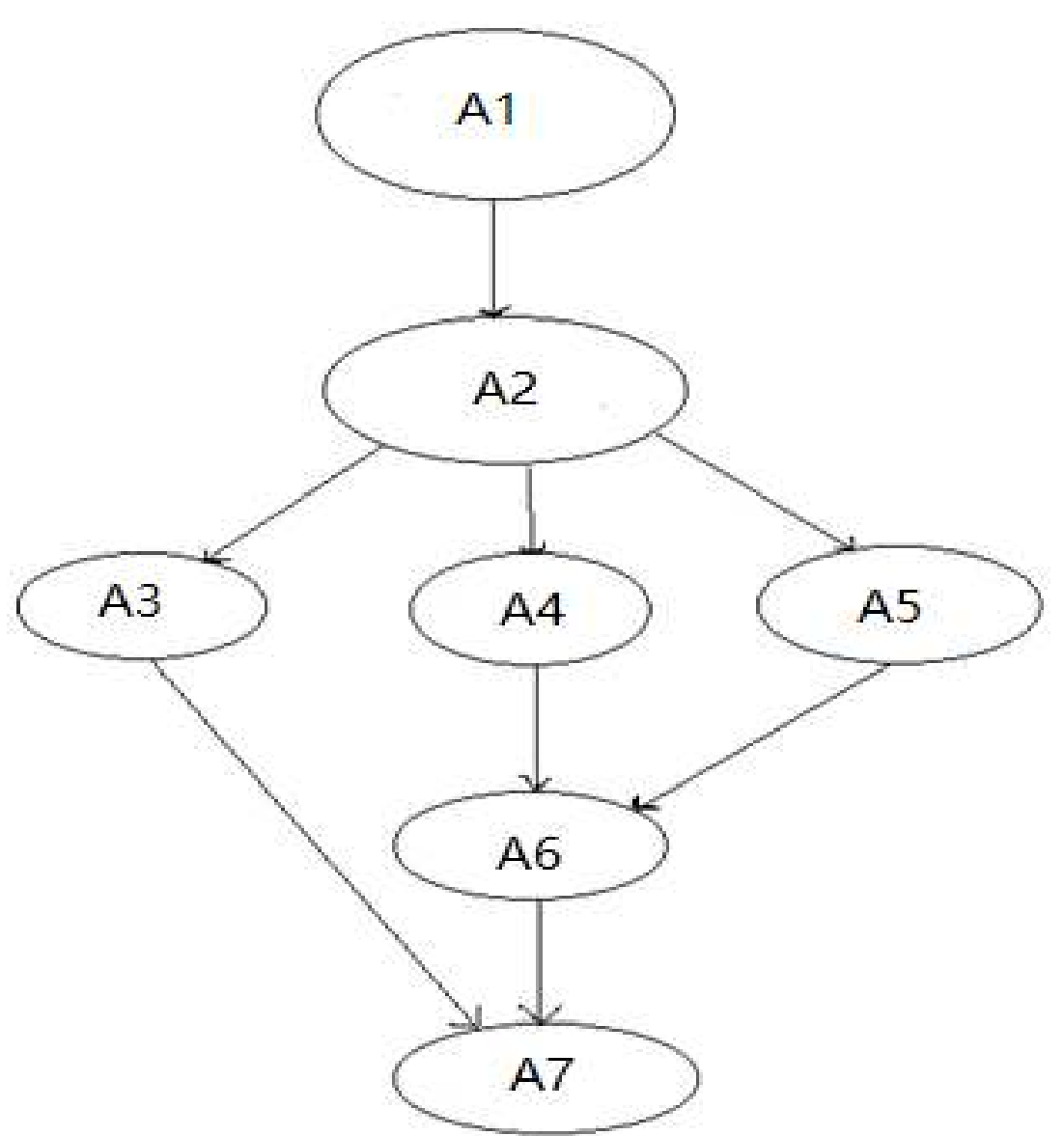

Figure1 The attribute hierarchy model of unior middle school MLD

We select 282 students (109 suspected mathematics learning disablity of students, and other four math scores in the class and grade evaluation low-ranking class's and grade's student 173 people) from four schools of Hebei and Liaoning province take the diagnostic test, we send 282 questionnaires in total and took back 255 questionnaires, after the questionnaire screen, we got 227 available questionnaire.

Table2 Index of two model

\begin{tabular}{|c|c|c|c|}
\hline & AIC & BIC & RMSEA \\
\hline DINA & 4697.64 & 4926.82 & 0.065 \\
\hline DINO & 4723.75 & 4952.92 & 0.054 \\
\hline
\end{tabular}

The results of the data analysis show that :(1) the attribute hierarchy model of junior middle school mathematics learning difficulty which was preliminarily established through literature analysis and expert evaluation method is reasonable fit $(\mathrm{RMSEA}=0.054)$; (2) based on the cognitive diagnosis theory, the diagnosis of students with mathematics learning disablity in middle school is basically in line with the expectation and the diagnostic accuracy is good (> 70\%). (3) in this study, children with mathematics learning difficulty can be divided into 9 types; (4) the diagnosis of the mathematics learning disablity children's show that their metacognitive ability deficit is mainly about insufficient strategy using. The subsequent remedial teaching should be mainly about metacognitive strategy training. The study also shows that it is feasible to use the cognitive diagnostic theory to establish diagnostic tests for children with difficulty in math learning.

Table3 number and proportion of each MLD style

\begin{tabular}{|c|c|c|}
\hline Pattern & Number & proportion \\
\hline 0000000 & 10 & $4.40 \%$ \\
\hline 1111010 & 5 & $2.20 \%$ \\
\hline 1111110 & 4 & $1.76 \%$ \\
\hline 1100111 & 4 & $1.76 \%$ \\
\hline 1110100 & 1 & $0.44 \%$ \\
\hline 1110010 & 3 & $1.32 \%$ \\
\hline 1110011 & 5 & $2.20 \%$ \\
\hline 1100111 & 4 & $1.76 \%$ \\
\hline 1110111 & 45 & $19.82 \%$ \\
\hline 1111111 & 113 & $49.78 \%$ \\
\hline
\end{tabular}

